\title{
The effect of Silk damping on primordial magnetic fields
}

\author{
Axel Brandenburg a,1, Kari Enqvist ${ }^{\mathrm{b}, 2}$, Poul Olesen ${ }^{\mathrm{c}, 3}$ \\ ${ }^{a}$ Department of Mathematics and Statistics, University of Newcastle upon Tyne, NE1 7RU, UK \\ ${ }^{\mathrm{b}}$ Department of Physics, University of Helsinki, FIN-00014 Helsinki, Finland \\ c The Niels Bohr Institute, University of Copenhagen, Blegdamsvej 17, DK-2100 Copenhagen, Denmark
}

Received 19 September 1996; revised manuscript received 18 November 1996

Editor: P.V. Landshoff

\begin{abstract}
We study the effects of plasma viscosity on the dynamics of primordial magnetic fields by simulating magnetohydrodynamics in the early universe by appropriate non-linear cascade models. We find numerically that even in the presence of large kinetic viscosity, magnetic energy is transferred to large length scales. There are indications, however, that the inverse cascade stops at a given time which depends on the magnitude of viscosity. For realistic viscosities we do not find equipartition between magnetic and kinetic energies.
\end{abstract}

There are many ways that magnetic fields could have been generated at various stages in the early universe. In the theory of galactic magnetism such fields are often referred to as "cosmological" or "primordial", because they are created well before galaxies are formed. These fields may play the role of an initial condition for the galactic dynamo, a mechanism that would amplify magnetic fields and convert kinetic energy into magnetic [1].

There are two major problems when invoking primordial magnetic fields as possible seed magnetic fields for the galactic dynamo. One problem is the very small length scale of such magnetic fields. The horizon scale at the time of the electroweak phase transition is just a few centimeters, corresponding to about $1 \mathrm{AU}$ at the present time. This is nine orders of magnitude shorter than the radius of typical galaxies.

\footnotetext{
${ }^{1}$ E-mail: Axel.Brandenburg@Newcastle.ac.uk.

${ }^{2}$ E-mail: enqvist@pcu.helsinki.fi.

${ }^{3}$ E-mail: polesen @nbi.dk.
}

However, this view is too simplistic, because nonlinear effects inherent in the magnetohydrodynamic (MHD) equations can lead to a redistribution of magnetic energy over different length scales. Unfortunately, MHD in $3+1$ exceeds the possibilities of present day computers. Therefore one has to resort to models which simulate the MHD equations. In a previous Paper [2], hereafter referred to as Paper 1, we developed a fully relativistic $3+1 \mathrm{~d}$ version of the socalled cascade model [3] appropriate for MHD and found that an inverse cascade is operative, whereby magnetic energy is continuously transferred to larger length scales. The other problem is that around the time of recombination photon diffusion becomes very large and could smooth out all fluctuations [4]. This may then also destroy the magnetic field [5]. The purpose of this Paper is to show that this too is too simplistic a viewpoint, and that nonlinear effects most likely prevent this from happening.

The basic equations have been presented and discussed in Paper 1. We started out from the fully rela- 
tivistic MHD equations in expanding (flat) space and showed that all the terms arising from the expansion can be removed by using rescaled quantities and conformal time,

$t=\int d t_{H} / R\left(t_{H}\right)$

where $t_{H}$ is the Hubble time and $R\left(t_{H}\right)$ is the expansion factor. Starting from random initial conditions, we obtained turbulent velocity and magnetic fields, very much like those in ordinary (nonrelativistic) decaying hydromagnetic turbulence. In order to study the effects of this kind of turbulence we adopted a simple cascade model that captures the qualitative features rclated to turbulent energy spectra. Such models [3] have been rather successful in predicting even subtle corrections to Kolmogorov turbulence due to intermittency effects [6]. (For a recent review, see Ref. [7].) Our model is more general in that it includes the effects of magnetic fields. Unlike nonmagnetic turbulence, in the presence of magnetic fields there is an inverse cascade of magnetic helicity, which leads to a transport of magnetic energy to larger and larger scales [8]. Using our cascade model (also called shell model) we found that the integral scale

$l_{0}=\int(2 \pi / k) E_{M}(k) d k / \int E_{M}(k) d k$,

where $E_{M}(k)$ is the magnetic energy spectrum, increases with the Hubble time approximately like $t_{H}^{0.25}$.

In Paper 1 we considered the case where the kinematic viscosity $\nu$ was equal to the magnetic diffusivity $\eta$ (inverse electrical conductivity) which, in turn, was assumed to be small (corresponding to a large magnetic Reynolds number). However, around the time of recombination the photon mean free path $\lambda_{y}$ became very large and photon diffusion became very efficient in smoothing out virtually all inhomogeneities of the photon-baryon plasma [9]. This process if often referred to as Silk damping, which corresponds to a kinematic viscosity $\nu \simeq \lambda_{\gamma}$ (in natural units). We have computed numerically the evolution of magnctic and kinetic energy spectra in two completely different cascade models and studied the effects of viscosity. Our results are presented in Figs. 1 and 2 and the main point can be summarized as follows: in the cascade models magnetic energy is transferred to large length scales even in the presence of large viscosity. It seems likely that the same is true also in full $3+1$ MHD.

Let us first use the cascade model of Paper 1 to investigate the effect of very large values of $\nu$ on the magnetic field. In this model velocity and magnetic fields are described by the variables $v_{n}$ and $b_{n}$, representing the "collective" behavior at wavenumber $k_{n}$. In the original model, $k_{n}=r^{n}$ with $r=2$. Below we shall also consider a continuous version of this model with $r \rightarrow 1$, which was originally studied by Parisi [10] in a hydrodynamical context. We reiterate here the salient features of the model. We present the equations of motion in a slightly different form with the negative and positive helicity states split, to facilitate comparison with the subsequent continuous model:

$$
\begin{aligned}
& \left(d v_{n}^{+} / d t+\nu k_{n}^{2} v_{n}^{+}\right)^{*}=i k_{n}\left(v_{n+1}^{-} v_{n+2}^{+}+\frac{1-r}{r^{2}} v_{n-1}^{-} v_{n+1}^{-}\right. \\
& \quad-\frac{1}{r^{3}} v_{n-1}^{-} v_{n-2}^{+}-b_{n+1}^{-} b_{n+2}^{+}-\frac{1-r}{r^{2}} b_{n-1}^{-} b_{n+1}^{-} \\
& \left.\quad+\frac{1}{r^{3}} b_{n-1}^{-} b_{n-2}^{+}\right)
\end{aligned}
$$

and

$$
\begin{aligned}
& \left(d b_{n}^{+} / d t+\eta k_{n}^{2} b_{n}^{+}\right)^{*}=i \frac{k_{n}}{r(1+r)}\left(v_{n+1}^{-} b_{n+2}^{+}\right. \\
& \quad-b_{n+1}^{-} v_{n+2}^{+}+v_{n-1}^{-} b_{n+1}^{-}-b_{n-1}^{-} v_{n+1}^{-}-v_{n-1}^{-} b_{n-2}^{+} \\
& \left.\quad+b_{n-1}^{-} v_{n-2}^{+}\right)
\end{aligned}
$$

with similar equations with $v_{n}^{+} \leftrightarrow v_{n}^{-}$and $b_{n}^{+} \leftrightarrow b_{n}^{-}$. The model describes an expanding radiation dominated magnetic universe $(p=p / 3)$. In Eqs. (3), (4) $t$ is the conformal timc, and the units are such that $\rho \sim T^{4}$ (for details, see [2]). Eqs. (3), (4) conserve energy and helicity in the absence of magnetic diffusion $\eta$ and plasma viscosity $\nu$, and + and - are related to magnetic helicity. Thus, if $\nu=\eta=0$, the quantities

$$
\begin{aligned}
& E=\sum\left(\left|b_{n}^{+}\right|^{2}+\left|b_{n}^{-}\right|^{2}+\left|u_{n}^{+}\right|^{2}+\left|u_{n}^{-}\right|^{2}\right), \\
& H=\sum\left(\left|b_{n}^{+}\right|^{2}-\left|b_{n}^{-}\right|^{2}\right) / k_{n},
\end{aligned}
$$

are conscrved. This is a direct consequence of Eqs. (3), (4). In the case of ordinary MHD $b$ is just the magnetic field. In a cosmological setting with a flat universe, $b$ is the magnetic field multiplied by $R(t)^{2}$, and $t$ is conformal time. The conservation of helicity exhibited above is nontrivial in three, but not 
in two dimensions, so that in this sense the cascade model is three dimensional.

There does not exist any proof that the cascade model and the standard MHD equations are equivalent. There are features which are similar in both cases: the equations couple many different scales (making it hard to predict anything a priori, especially when the Reynolds number is large and nonlinear effects are important), they have similar conservation laws, and the equations of motion are similar. Also, in the pure hydrodynamic case the cascade model equations have been compared to experiments [6] and good results on intermittency have been obtained. In any case, the properties of turbulence have not been derived from first principles, and the cascade model is therefore an interesting (toy?) tool.

For large diffusion coefficients the equations become stiff and it is therefore essential to solve for the diffusion term exactly. Using the identity

$\frac{d v_{n}}{d t}+\nu k_{n}^{2} v_{n}=e^{-\nu k_{n}^{2} t} \frac{d}{d t}\left(v_{n} e^{\nu k_{n}^{2} t}\right)$

we solve equations of the form

$\frac{d v_{n}}{d t}+\nu k_{n}^{2} v_{n}=N_{n}(t)$

by a modified second order Adams-Bashforth scheme

$$
\begin{aligned}
& v_{n}(t+\delta t)=K_{n}\left\{v_{n}(t)\right. \\
& \left.+\frac{1}{2} \delta t\left[3 N_{n}(t)-K_{n} N(t-\delta t)\right]\right\}
\end{aligned}
$$

where

$K_{n}=e^{-\nu \delta t k_{n}^{2}}$.

We start from an initial condition that yields a magnetic energy spectrum similar to that found at later stages. Fig. 2 of Paper 1 suggests that the spectrum has developed an inertial range which is approximately constant, $E_{M}(k)=$ const. for $k_{d}<k<k_{0}$, where $k_{0}=2 \pi / l_{0}$ is the wavenumber corresponding to the integral scale and $k_{d} \gg k_{0}$ the wavenumber of the diffusive cutoff scale. We adopt 30 wavenumber shells ( $1<n<N$ with $N=30$ ) and place the cutoff wavenumber at $n=27$ and use $\eta=10^{-11}$. We put $v_{n}=0$ initially and compare the results for two different values of $\nu\left(10^{-2}\right.$ and $\left.10^{2}\right)$.

The resulting spectra are displayed in Fig. 1. The results show that $v_{n}$ is generated from $b_{n}$, but it gets weaker if $\nu$ is increased. At small $\nu$ and at large times there is an approximate equipartition of the magnetic and kinetic energies with $v_{n}=b_{n}=B_{n} R^{2}$, where $B_{n}$ is the unscaled magnetic field. Since we are using units in which the initial effective energy density $\rho+p=4 \rho / 3=1$ it follows that $v_{n}$ is the Alfven velocity $B_{n} / \sqrt{\frac{4}{3} \rho}$ of the equilibrium plasma. For large $\nu$ equipartition is lost, which signals the breakdown of the perturbative approach. As we shall argue later, the large $\nu$ case is appropriate for the very early universe. More importantly, Fig. 1 shows that the evolution of $b_{n}$ is not affected by $v_{n}$ and $\nu$, but is rather governed by ohmic decay, $b_{n} \sim \exp \left(-\eta k_{n}^{2} t\right)$. It may also be seen that in the case of large kinetic viscosity $\nu=100$ the velocity modes decay approximately according to $v_{n} \sim \exp \left(-\nu k_{n}^{2} t\right)$.

We shall now study the effect of a large $\nu$ on $b$. We have done some more detailed calculations in order to investigate what is behind the behaviour shown in Fig. 1. The results are shown in Fig. 2, which shows that for a sufficiently large viscosity, the inverse cascade stops. With a magnetic energy of $10^{-8}$, the cascade goes over five decades in the most unfavourable case $\nu=100$. The inverse cascade stops at some low value of $\mathrm{k}$, and the field just disappears by Ohmic decay. One can then ask, at which temperature does the inverse cascade stop? To estimate this, one studies the ratio between the non-linear terms and the Silk viscosity term, i.e. the Reynolds number. We start at the annihilation $t_{H} \sim 1 \mathrm{sec}$, where we assume that there are no velocity fluctuations. This is not true if the primordial field originates at the electroweak phase transition $T \sim 100 \mathrm{GeV}$, since there will always be velocity fluctuations associated with the magnetic field. However, to allow for the optimal conditions for Silk diffusion, we assume that there are no velocity fluctuations at the annihilation. We then have to estimate the resulting Reynolds number,

$$
\operatorname{Re} \sim \frac{v}{k \nu_{\mathrm{Silk}} / R},
$$

where the viscosity $\nu_{\text {Sik }} / R$ is effectively given by the photon diffusion length,

$\nu \sim \frac{10^{6}}{T^{3}} \mathrm{GeV}^{2}$.

As mentioned above, the inverse cascade spans over 

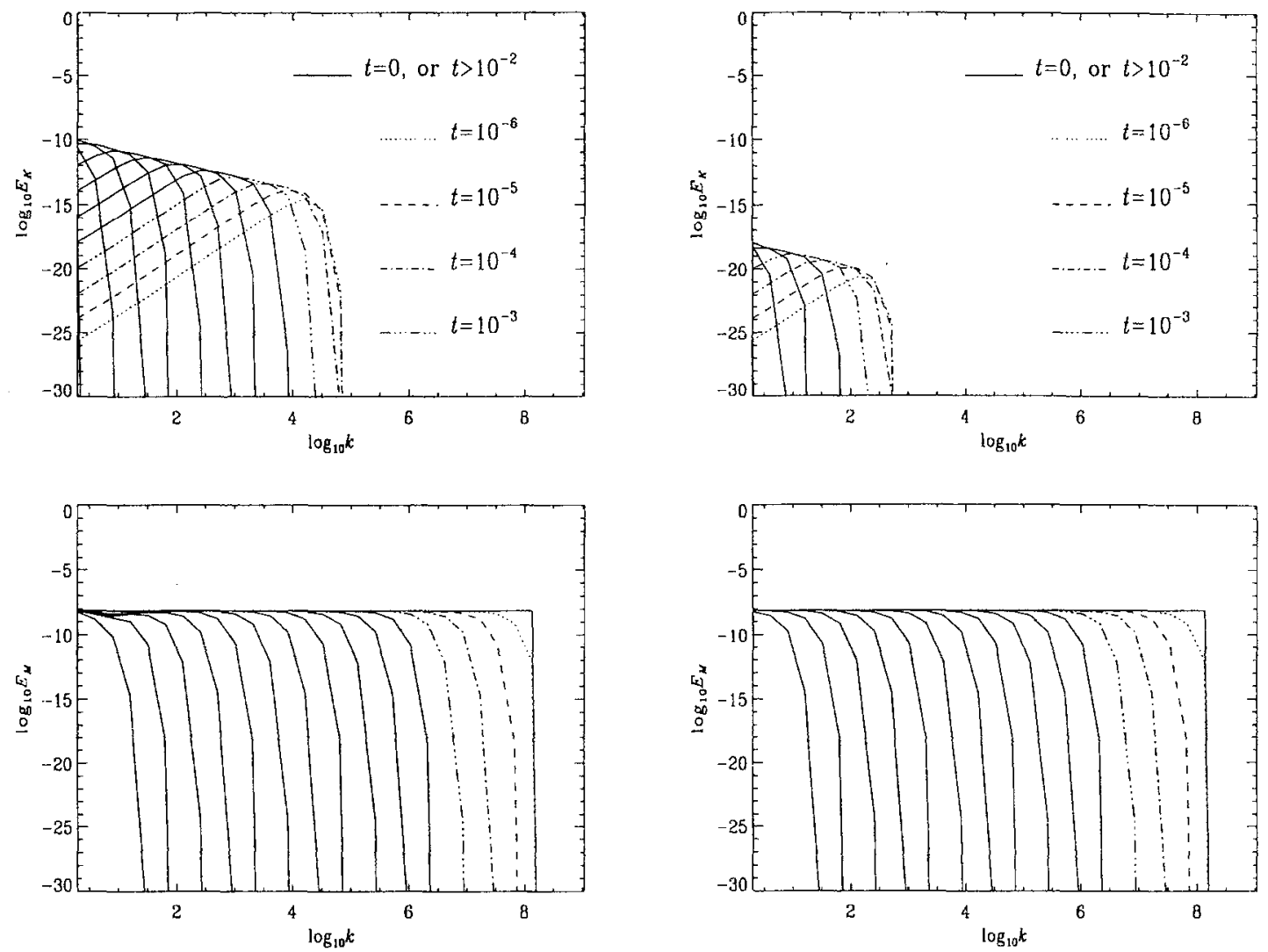

Fig. 1. Magnetic and plasma kinetic energy spectrum as a function of the wave number $k$ in the cascade model for small $\left(\nu=10^{-2}\right.$ ) plasma viscosity (left) and large $\left(\nu=10^{2}\right)$ plasma viscosity (right). The highest time is $t=10^{8}$, corresponding to a Hubble time $10^{16}$.

five decades in $k$. Thus, the typical $k$ which enters in $\operatorname{Re}$ in Eq. (10) is given by

$k=10^{-5} / x t_{H}^{\mathrm{ann}}$,

where $x t_{H}^{\text {ann }}$ is the spatial scale scale of the magnetic field at the annihilation. The fraction $x$ is thus determined by the mechanism for producing the primordial field. Before annihilation, we assume that the viscosity is so unimportant that it can be ignored. This is why we start at annihilation, where Silk diffusion becomes very large. The inverse cascade stops when $R e$ is of order one, i.e. when the diffusion equals the nonlinear terms. We may therefore conclude that in the particular case displayed in Fig. $2(\nu=100)$, with $v=10^{-4}$, the cascade stops close to recombination.

To verify that the above results are generic and not just a feature of the model adopted, we will now consider another model of the full $3+1$ MHD [10]. For- mally it may be obtaincd from (3), (4) by passing to the limit $r \rightarrow 1$, but it may also be viewed as a completely independent model of MHD. Writing $r=1+\epsilon$ we get

$$
\begin{gathered}
\left(\frac{\partial v^{-1}(k, t)}{\partial \epsilon t}+\nu k_{n}^{2} v^{+}(k, t)\right)^{*}=i k\left(4 v^{-} k \frac{\partial v^{+}}{\partial k}\right. \\
+2 v^{+} k \frac{\partial v^{-}}{\partial k}+3 v^{-} v^{+}-\left(v^{-}\right)^{2}-4 b^{-} k \frac{\partial b^{+}}{\partial k} \\
\left.-2 b^{+} k \frac{\partial b^{-}}{\partial k}-3 b^{+} b^{-}+\left(b^{+}\right)^{2}\right)+\mathcal{O}(\epsilon)
\end{gathered}
$$

and

$$
\begin{aligned}
& \left(\frac{\partial b^{+}(k, t)}{\partial \epsilon t}+\eta k_{n}^{2} b^{+}(k, t)\right)^{*}=i k\left(b^{+} k \frac{\partial v^{-}}{\partial k}\right. \\
& \quad+2 v^{-} k \frac{\partial b^{+}}{\partial k}-v^{+} k \frac{\partial b^{-}}{\partial k}-2 b^{-} k \frac{\partial v^{+}}{\partial k}+v^{-} k \frac{\partial b^{-}}{\partial k}
\end{aligned}
$$



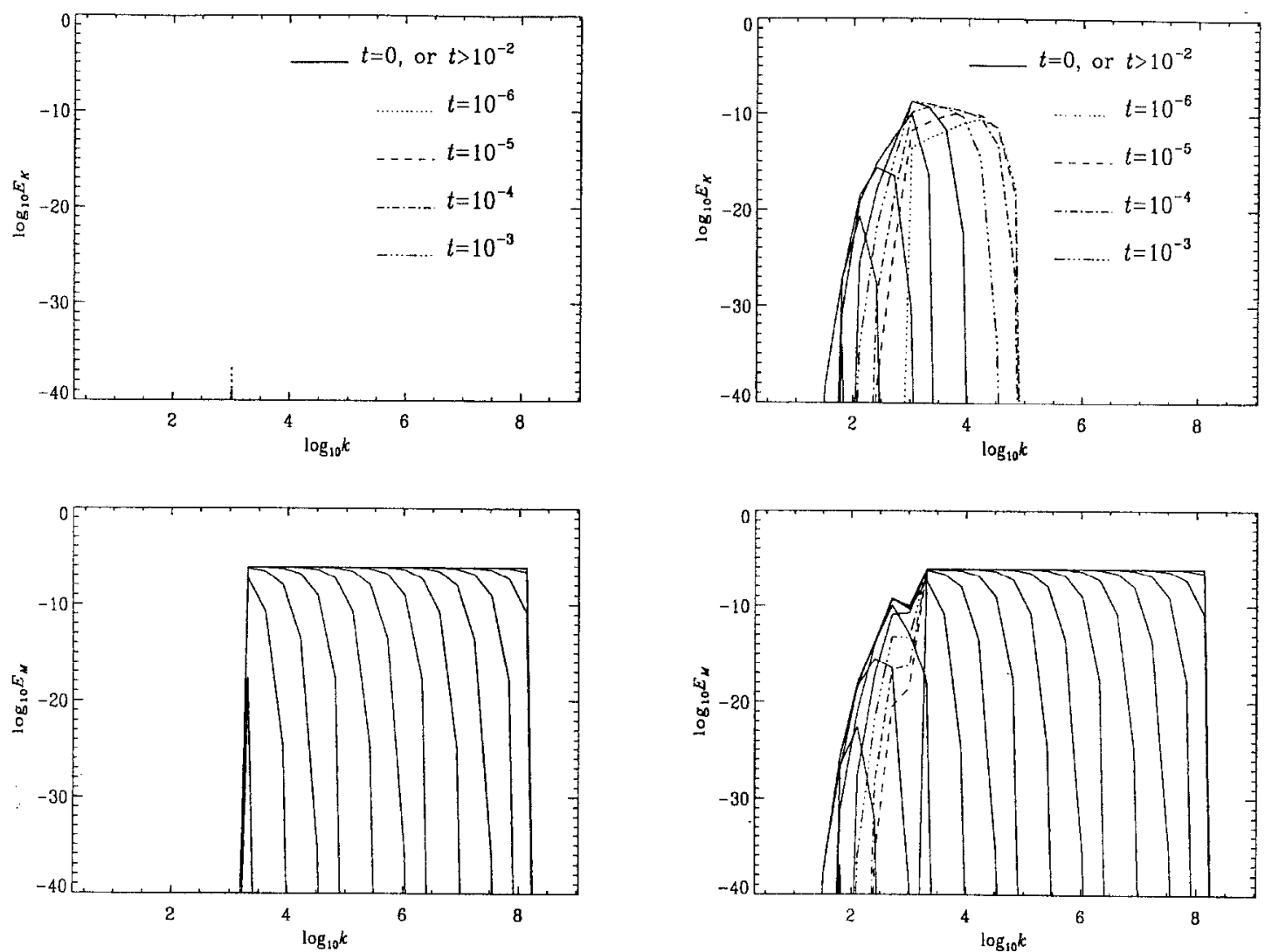

Fig. 2. The behaviour of the cascade model for $\nu=10^{2}$ (left) and $\nu=10^{-2}$ (right) at large times (the last time slice is $t=10^{37}$ ). The figure shows what happens to an initially flat spectrum (which does not extend to $k=0$ ). Here $\eta=10^{-31}$.

$$
\left.-b^{-} k \frac{\partial v^{-}}{\partial k}\right)+\mathcal{O}(\epsilon)
$$

In addition to Eqs. (13) and (14) there are two equations more, obtained by making the replacements $b^{+} \leftrightarrow b^{-}$and $v^{+} \leftrightarrow v^{-}$. These equations conserve the quantities

$E=\int \frac{d k}{k}\left(\left|v^{+}\right|^{2}+\left|v^{-}\right|^{2}+\left|b^{+}\right|^{2}+\left|b^{-}\right|^{2}\right)$,

$H=\int \frac{d k}{k^{2}}\left(\left|b^{+}\right|^{2}-\left|b^{-}\right|^{2}\right)$,

for $\nu=\eta=0$, provided the following boundary conditions are satisfied for $k \rightarrow 0$ and $k \rightarrow \infty$,

$k v^{-}\left(v^{+}\right)^{2} \rightarrow 0, k v^{+} b^{+} b^{-} \rightarrow 0, k v^{-} b^{+} \rightarrow 0$,

$k v^{+}\left(v^{-}\right)^{2} \rightarrow 0, k v^{-} b^{+} b^{-} \rightarrow 0, k v^{+}\left(b^{-}\right)^{2} \rightarrow 0$, and

$$
\begin{aligned}
& v^{-}\left(b^{+}\right)^{2} \rightarrow 0, v^{+}\left(b^{-}\right)^{2} \rightarrow 0, \\
& b^{+} b^{-} v^{+} \rightarrow 0, \quad b^{+} b^{-} v^{-} \rightarrow 0
\end{aligned}
$$

respectively. If the conditions at infinity are not satisfied, this corresponds to "diffusion at infinity".

We shall now simplify the continuous cascade model by using a scaling first introduced by Parisi [10] for the case of pure hydrodynamics without helicity conservation. If one considers the original discrete equations ( 3 ), (4) in the absence of viscosity, and assume that we start at time $t=0$ with a primordial spectrum

$b^{+}(k, 0)=b^{-}(k, 0)=k^{p}$,

$v^{+}(k, 0)=v^{-}(k, 0)=0$, 
then it is easy to see that the discrete cascade model equations have a solution with the scaling form

$b^{+}(k, t)=k^{p} B\left(k^{1+p} t\right), \quad b^{-}(k, t)=k^{p} M\left(k^{1+p} t\right)$,

$v^{+}(k, t)=k^{p} u\left(k^{1+p} t\right), \quad v^{-}(k, t)=k^{p} m\left(k^{1+p} t\right)$.

These equations imply that $E_{B} / k^{2 p-1}$ is a function of $k^{p+1} t$ only.

If we insert the scaling (19) in the continuous cascade model Eqs. (13), (14), then we see that powers of $k$ cancel out neatly, and we are left with the coupled equations ${ }^{4}$

$$
\begin{aligned}
& -u^{\prime}(x)=3(2 p+1) u m+4(1+p) x m u^{\prime} \\
& +2(1+p) x u m^{\prime}-m^{2}-3(1+2 p) M B \\
& -4(1+p) x M B^{\prime}-2(1+p) x B M^{\prime}+M^{2},
\end{aligned}
$$

and

$$
\begin{aligned}
& -B^{\prime}(x)=3 p m B-3 p u M+2(1+p) x m B^{\prime} \\
& +(1+p) x B m^{\prime}-(1+p) x u M^{\prime}-2(1+p) x M u^{\prime} \\
& +(1+p) x m M^{\prime}-(1+p) x M m^{\prime},
\end{aligned}
$$

as well as two equations where the interchanges $B \leftrightarrow$ $M$ and $u \leftrightarrow m$ have been made. Thus, the original set of $2 N$ (with $n \leq 2 N$ ) coupled differential equations have been replaced by only four. In these equations

$x=k^{1+p} t$

is the scaling variable.

It should be emphasized that the continuous cascade model is a priori "as good as" the discrete one, since in both cases the conservation equations are satisfied when there is no diffusion (except for possible diffusion at infinity). It may of course turn out that phenomenologically one type is better than the other.

In Paper 1 we found that if one starts with a primordial spectrum $p=3 / 2$, then there is an inverse cascade, transferring energy from large to small $k$. The scaling equtions (19) satisfy this: For a fixed value of the variable $x$ in (22), the functions $B, M, u$, and $m$ have some definite values. Thus, as time is increased,

\footnotetext{
${ }^{4}$ We have here selected the phase of $b$ and $v$ to be $-\pi / 2$. With a dynamical phase the number of equations double from four to eight, since each $b$ and $v$ have a real and imaginary part.
}

these values remain the same if $k$ is diminished in such a way that $x$ remains constant. Thus, the scaling relations (19) predicts an inverse cascade.

This is also seen if we consider the integral scale (the "correlation length"), given by Eq. (2), which measures the large scale structures in comoving coordinates. We may consider $I_{0}$ as the expectation value of $1 / k$. From scaling we therefore expect that

$l_{0} \approx t^{1 /(1+p)} \approx t_{H}^{1 / 2(1+p)}$,

where $k^{p}$ is the initial primordial spectrum. From this we see that for $p=3 / 2$ one obtains

$l_{0} \approx t_{H}^{0.2}$,

where the power is in essential agreement with the previously found [2] value $p \approx 0.25$.

Since we do not commit ourselves to any specific model, let us consider an initial spectrum $k^{p}$. The integral scale then behaves as in (23) in comoving coordinates, which means that in physical coordinates it goes like

$l_{0}^{\text {physical }} \approx t_{H}^{\frac{2+p}{2(1+p)}}$.

Thus, if there exists a model with $p=0$, in such a case the large scale structures are of the order the horizon. The case $p=0$ corresponds to a scale invariant primordial spectrum $d k / k$.

The scaling relation is in general only valid in the "incrtial range" where viscosity can be ignored, because the viscosity term is inconsistent with the scaling. There is, however, one exception, where scaling and viscosity are consistent, namely $p=1$. Here $-u^{\prime}(x)$ on the left hand side of Eq. (20) is replaced by

$-u^{\prime}(x)-\nu u(x)$

Similarly, in Eq. (21) $-B^{\prime}(x)$ is replaced by

$-B^{\prime}(x)-\eta B(x)$.

This is the reason we have preferred to perform the numerical calculations in the scaled version of the continuous cascade model for the case $p=1$. We took as the initial conditions $u=m=0, M=B=1$ and as in the discrete case, considered two viscosities $\left(\nu=10^{-2}\right.$ and $\left.\nu=10^{2}\right)$. The units are again such 
(a)

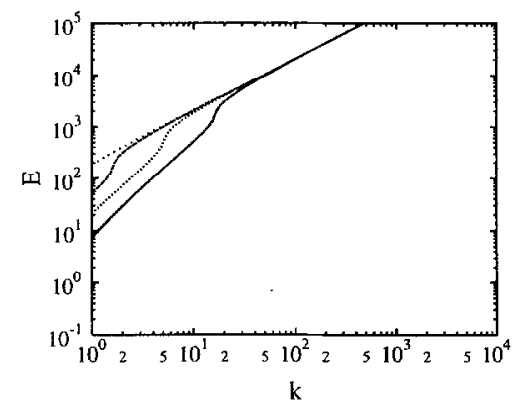

(b)

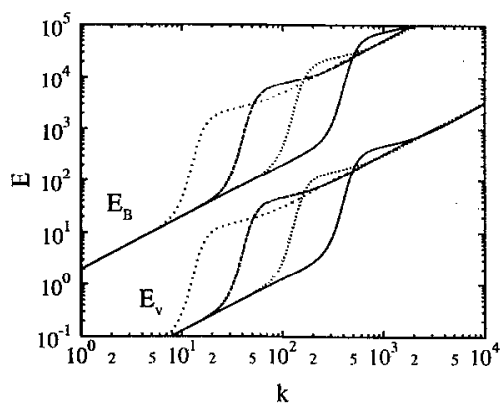

Fig. 3. The energy spectra in the continuous cascade model for (a) small ( $\nu=10^{-2}$ ) plasma viscosity; in this case there is equipartition and magnetic and kinetic energies are equal; (b) large $\left(\nu=10^{2}\right)$ plasma viscosity; absence of equipartition is apparent. In both figures the curves are, from right to left, for conformal times $t=1,10,100$ and 1000 , and $\eta=10^{-9}$.

that initially $\rho=\rho_{0}=1$. Magnetic diffusion was set to $\eta=10^{-9}$. The difference with respect to the discrete case is the shape of the initial spectrum of $b_{n}$. In the discrete case $b_{n}$ was initially given by $\sqrt{k}$, whereas here we took $b_{n}(k, 0)=k$. The results are depicted in Fig. 3, where the inverse cascade can clearly be seen, virtually independent of viscosity. They also confirm that equipartition is lost at large $\nu$.

We have also checked the scaling of $b$ for the data presented in Paper 1 . This corresponds to $p=3 / 2$. The scaling variable is thus $x=k^{5 / 2} t$, where it is important that $t$ is the conformal time; in terms of the Hubble time, the scaling variable is $k t_{H}^{0.2}$. From its derivation, scaling is only valid in this case in the inertial range, where viscosity can be ignored. We see that within the fluctuations of the raw data, the predictions are approximately valid for large times (see Fig. 4).

Our results very much suggest that in the real MHD, inverse cascade is operative and is essentially not affected by Silk damping, except very late and perhaps for very weak fields (which anyhow are not interesting). In our units, in the early universe $\nu \gg 1$. Thus we may conclude that it is unlikely that there is equipartition in the very early universe. Our relativistic approach remains valid roughly until recombination, after which the plasma becomes matter dominated. (In the non-relativistic regime $\rho \sim R^{-3}$, which effectively produces an extra term of the form $-(\dot{R} / R) v$ in the equations of motion. However, because photon diffusion is so large, this braking due to expansion is unimportant and conclusions presented above still hold.)

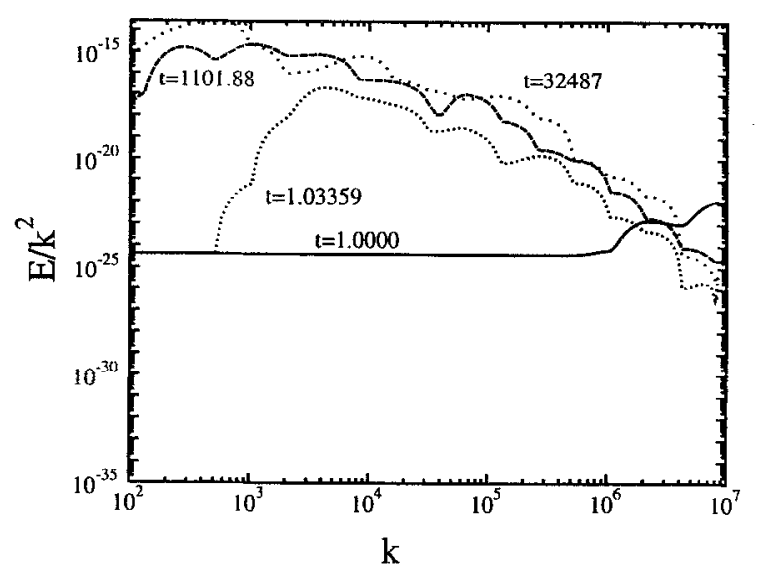

Fig. 4. The scaled energy spectrum of the discrete cascade model at different times (neglecting viscosity).

Therefore, it seems plausible that small random magnetic domains of the very early universe may grow to large scale fields, irrespective of the Silk diffusion.

\section{References}

[1] P.J.E. Peebles, The Large Scale Structure of the Universe (Princeton University Press 1980);

I. Wasserman, Astrophys. J. 224 (1978) 337;

E. Harrison, Mon. Not. R. Astr. Soc. 147 (1970) 279; 165 (1973) 185 ;

for a recent review, see R. Beck, A. Brandenburg, D. Moss, A. Shukurov, and D. Sokoloff, Ann. Rev. Astron. Astrophys. 34 (1996) 153.

[2] A. Brandenburg, K. Enquist and P. Olesen, Phys. Rev. D 54 (1996) 1291. 
[3] E.B. Gledzer, Sov. Phys. Dokl. 18 (1973) 216;

K. Ohkitani and M. Yamada, Progr. Theor. Phys. 81 (1989) 329.

[4] J. Silk, Astrophys. J. 151 (1968) 459.

[5] K. Jedamzik, V. Katalinic and A. Olinto, astro-ph/9606080.

[6] M.H. Jensen, G. Paladin and A. Vulpiani, Phys. Rev. A 43 (1991) 798.

「7ๆ T. Bohr, M.H. Jensen, G. Paladin and A. Vulpiani, Dynamical Systems Approach to Turbulence, Cambridge
Nonlinear Science Series (Cambridge University Press, to be published) (1996).

|8| A. Pouquet, U. Frisch and J. Léorat, J. Fluid Mech. 77 (1976) 321.

[9] E.W. Kolb and M. Turner, The Early Universe (AddisonWesley, New York 1990).

[10] G. Parisi, Rome preprint ROM2F-90/37, unpublished (1990). 